\title{
Total Lagrangian SPH Modelling of Necking and Fracture in Electromagnetically Driven Rings
}

\author{
T. De Vuyst · R. Vignjevic
}

Received: date / Accepted: date

\begin{abstract}
This paper describes research on the prediction of necking and failure in metals at very high strain rates. The model developed in this paper uses a total Lagrangian SPH formulation with a normalised kernel. The detailed data from electromagnetically driven ring experiments by Zhang and Ravi-Chandar (2006) is used to evaluate the accuracy of the model predictions. In order to correctly model fracture in the total Lagrangian SPH formulation a visibility criterion based on a truncated cone has been implemented to remove particles obscured by a failed particle. A Johnson-Cook plasticity model is used in combination with a Lemaitre damage model to describe the plastic deformation and fracture of the rings. The effect of Joule heating due to the current induced in the ring is taken into account in the constitutive model. The acceleration due to the ring currents was implemented in the SPH code as a body force. The results demonstrate that this type of model is capable of predicting the number of fragments as well as the time of fracture. In agreement with experimental data, the model also predicts arrested necks and bending in the fragments.
\end{abstract}

T. De Vuyst

Crashworthiness, Impact and Structural Mechanics Group, School of Engineering, Cranfield University, Cranfield, MK43 OAL, UK

Tel.: +44-1234-758286

Fax: +44-1234-758217

E-mail: t.devuyst@cranfield.ac.uk

R. Vignjevic

Crashworthiness, Impact and Structural Mechanics Group, School of Engineering, Cranfield University, Cranfield, MK43 $0 \mathrm{AL}, \mathrm{UK}$

Tel.: +44-1234-754736

Fax: +44-1234-758207

E-mail: v.rade@cranfield.ac.uk
Keywords smoothed particle hydrodynamics . meshless · modelling · fracture · expanding ring · necking $\cdot$ strain-rate

\section{Introduction}

Due to their potential in dealing with fracture, meshless methods, such as Smoothed Particle Hydrodynamics $(\mathrm{SPH})$, are of particular interest for the prediction of fragmentation and fracture at high strain rates. This paper describes research on the numerical prediction of necking and fracture of electromagnetically driven rings using the SPH method. In an electromagnetically driven ring experiment a metal ring is radially accelerated by an electromagnetic force acting on the ring. These electromagnetic forces are generated by inducing a current in the ring by discharging a capacitor into a coil located inside the ring. A schematic representation of this setup is shown in Figure 1. There are a number of publications with detailed experimental results as well as numerical models of electromagnetically driven expanding ring fragmentation. In the following paragraphs a brief overview is provided of the most relevant papers.

\subsection{Electromagnetically Driven Ring Experiments}

A widely cited paper on electromatically driven expanding ring experiments is by Grady and Benson (1983). This paper describes an electromagnetical expanding ring test method in detail and presents results for copper and aluminium rings. They show the influence of ring velocity on fracture strain and the dependence of fragment number on kinetic energy. The data in this paper is frequently used to validate numerical models of 
ring fragmentation. Satapathy and Landen (2006) also describe an electromagnetically driven expanding ring experiment. They study the effect of adiabatic heating, due to the current induced in the rings, on the mechanical properties during expansion, and compare this with equivalent isothermal properties. The paper does not discuss fragmentation of the rings. In a second paper (Landen et al., 2009) they extend their experiments to discuss the effect of (rapid) preheating on the mechanical properties and their effect on expansion of the rings. A good review of fragmentation models and experiments can be found in the paper by Zhang and Ravi-Chandar (2009c). One of the conclusions in this paper is that for fragmentation to be captured appropriately models must account both for damage localization and for statistical variations in this localization. The series of articles by Zhang and Ravi-Chandar (Zhang and Ravi-Chandar, 2006, 2008, 2009a,b) provides a large amount of data on ring fragmentation experiments. In Zhang and Ravi-Chandar (2006) they test Al 6061-0 specimens. The rings have a thickness of $0.5 \mathrm{~mm}$, length of $1.0 \mathrm{~mm}$ and a mean radius of $15.5 \mathrm{~mm}$. Expansion speeds were in a range of 80 to $200 \mathrm{~m} / \mathrm{s}$. Their analysis of neck development and fragment distribution lead them to conclude that the spacing between the necks and fragment length is determined by the presence of material imperfections and the propagation of Mott release waves. Based on the measured currents in the ring they calculate the temperature increase in the ring. Depending on the expansion speed the ring reached temperatures of $300-400^{\circ} \mathrm{C}$. This means that it is important to take into account the effect of temperature on the mechanical properties in numerical modelling of the process. It is shown (Zhang and Ravi-Chandar, 2006) that the avarage strain in the fragments stays below the Considère strain, and that the apparent increase of ductility at high strain rates, reported in other papers, is due to the measurement method which includes the higher strains in arrested necks within the fragments in the average strain of the fragment. The strain in the fragments (excluding necks) they measured were of the order of 0.22 , which is very close the static failure strain of 0.25 . The speed at which a localised deformation/damage spreads across the whole ring or cylinder section (and hence developes in a fracture) is identified as important (Zhang and Ravi-Chandar, 2006, 2009b). Therefore an appropreate 3D model must have sufficient mesh resolution across a section of the ring. If the mesh resolution is insufficient, or a $1 \mathrm{D}$ analysis is used localisation extends across the whole section of the ring to0 fast (in the case of single element cross section instantaneously). In Zhang and Ravi-Chandar (2009b) they stated that for quantitative predictions of the fragment distribution, it is necessary to develop better models of the process of ductile failure that occurs within the neck (or shear localized) regions.

\subsection{Electromagnetically Driven Ring Models}

Several models of the electromagnetically driven ring experiment have been published. Most models use Lagrangian FE codes (Sørensen and Freund, 2000; Pandolfi et al., 1999; Becker, 2002; Rusinek and Zaera, 2007; Zhang and Ravi-Chandar, 2008, 2009a), although sometimes Eulerian hydrocodes (Meulbroek et al., 2008) or finite difference models (Hu and Daehn, 1996; Johnson, 1981) are also used. The fragmentation stage is not always modelled with models restricting themselves to predicting the expansion speed and strain rate. A number of one dimensional models has been developed. Johnson (1981) proposed a 1D model for expanding ring fragmentation based on void growth and used an initial random distribution of porosity (imperfection). This 1D model was solved for a known strain rate history using the finite difference method. Hu and Daehn (1996) also presented a 1D finite difference model for dynamic tensile and expanding ring tests. They used a Holomontype material law with power law strain rate sensitivity. They analysed the effect of velocity, and parameters of the constitutive law (strain hardening exponent and strain rate sensitivity exponent) on ductility in tensile and expanding ring tests. The results compare well with experiment data from the literature. Zhou, Molinari and Ramesh use cohesive elements in a $1 \mathrm{D}$ model to analyse the fragment size distribution in radially expanding rings of brittle material (Zhou et al., 2006a) and elasto-visco-plastic material (Zhou et al., 2006b). Mercier and Molinari (2004) developed a linear perturbation analysis for the radial expansion of rings. They studied the effect of strain rate sensitivity and strain hardening on fragment size. Fyfe and Rajendran (1979) used perturbation analysis to study the effect of rate sensitivity and inertia effects on the strain at onset of necking. Triantafyllidis and Waldenmyer (2004) studied the effect of variations in strain hardening, strain rate sensitivity and temperature sensitivity on onset of necking strain using an analytical model. The model considered the coupled electromagnetic and thermomechanical problem. Shenoy and Freund (1999) studied the effect of expansion speed on the number of necks and they also concluded from their perturbation analysis that the number of necks is independent of the strain hardening exponent. Guduru and Freund (2002) analyse the effect of expansion speed on the number of necks and bifurcation strain. More recently a linear perturbation analysis of necking in hemispherical shells 
(Mercier et al., 2010) has been performed. This work also showed the influence of the constitutive law on the predicted number of necks. Vadillo et al. (2012) performed a linear perturbation analysis to study the effect of strain rate and strain rate sensitivity on localisation in expanding rings.

Sørensen and Freund (2000) presented a 2D finite element model of an expanding ring. The study focussed on the development of necking, and fragmentation of ductile materials. They used a Gurson model to represent void growth. The rings were loaded by applying a sinusoidal body force pulse to represent the electromagnetic force acting on the ring. Imperfections in their model were introduced as variations in ring thickness. The necking was shown to be quite sensitive to the perturbation of the ring's thickness. This lead to the conclusion that this type of perturbation is not appropriate for the prediction of fragment number and size. Becker (2002) presented 3D finite element modelling results of electromagnetically driven expanding ring experiments. The loading was applied by prescribing the radial velocity of the ring. Damage was modelled with a Gurson model. Material failure was determined with the Drucker stability criterion and a bifurcation model. Realistic strain to fracture and number of fragments were obtained, but no comparison with experimental data was made. Pandolfi et al. (1999) simulated the expanding ring experiments presented by Grady and Benson (1983). The simulation model in their paper used cohesive elements to model fracture. They demonstrated the ability of their method to model fragment number and size by comparison to the experiments of Grady and Benson (1983). However in their model only one element through the thickness of the ring was used. Consequently, as an element fails it immediately generates a fully fractured ring and release waves start propagating from the free surface. The same mesh approach is used by Rusinek and Zaera (2007). They use the Rusinek-Klepaczko constitutive equation and focus on the effect of the strain hardening, thermal softening and strain rate sensitivity terms in the constitutive equation, and the effect of Critical Impact Velocity on fragment size. Meulbroek et al. (2008) used CTH to simulate expanding ring fragmentation of Zhang and Ravi-Chandar (2006). Their model combined a 2D rectangular mesh with Bammann-Chiesa-Johnson constitutive law and Mie-Gruneisen equation of state. This constitutive model includes a void growth damage model. Due to limitations of the CTH software the loading of the ring was modelled by applying an initial velocity. For the same reason no random distribution of initial defects was applied. The model had problems with convergence and voids developed unrealistically early on in the simulation. The authors of this paper concluded that in order to predict the correct average fragment size and distribution, and the correct failure mechanism it was necessary to accurately model the necking mechanism (for the rings). Also the convergence issue they identified with the use of Eulerian codes like CTH needs to be resolved. Randles and Libersky (1996) were the first to apply the conventional SPH method to simulate fragmentation of explosively driven rings and cylinders. Their results show a very good correlation in terms of fragment mass statistics. Apart from Randles and Libersky's work, Hiroe et al. (2008) used 2D SPH in combination with Mott's stochastic failure model to assess 22 different explosively driven cylinder test configurations. Rabczuk and Belytschko (2004b) presented 2D cylinder fragmentation using the Element Free Galerkin Method (EFGM). In a recent paper by Dong et al. (2008) the Reproducing Kernel Particle Method (RKPM) was used to study failure of pressurised cylinders with an inital crack present. The pressure loading in this model was either applied as a step function at the beginning of the simulation or increased linearly over time. Note that this type of loading is different from the impulsive loading in explosively or electromagnetically driven cylinders. The work presented in this paper is an attempt to demonstrate that the SPH method in combination with a suitable material model is capable of quantitatively predicting fragmentation (fragment distributions and fracture time) in expanding ring experiments. The expanding ring experiments by Zhang and Ravi-Chandar (Zhang and RaviChandar, 2006) were used to validate the model accuracy.

\section{Description of Electromagnetically Driven Ring Experiments}

As already stated, the experimental data in Zhang and Ravi-Chandar (2006) was used to evaluate the accuracy of the model predictions. In these experiments electromagnetic induction was used to accelerate aluminium 6061-0 rings. A high speed camera with combined framing and streak imaging capability was used to provide detailed information about the necking and fracture of these rings. The experimental setup used is shown in Figure 1. In this figure the voltage source $\mathrm{V}$ is in reality a high voltage condensor with high capacitance (of the order of $\mu \mathrm{F}$ ). When the switch is closed a current $I_{1}$ runs through the coil, around which the ring is placed. The resistance of this primary circuit is $R_{1}$ and the inductance of the coil is $L_{11}$. The current pulse induces a current $I_{2}$ in the ring (which has resistance $R_{2}$ and $L_{22}$ ). The mutual inductance between the two coils is 


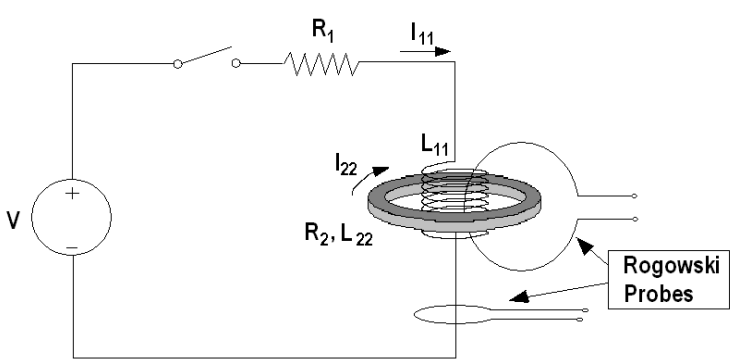

Fig. 1 Experimental Setup used by Zhang and RaviChandar (Zhang and Ravi-Chandar, 2006)

$L_{21}$. Both currents $I_{1}$ and $I_{2}$ are measured by Rogowski probes. The rings had a thickness of $0.5 \mathrm{~mm}$, length of $1.0 \mathrm{~mm}$ and a mean radius of $15.5 \mathrm{~mm}$. The high voltage condensor is charged with voltages of $5,6,7$ and $8 \mathrm{kV}$. This resulted in radial expansion speeds ranging from 80 to $200 \mathrm{~m} / \mathrm{s}$.

\section{Conventional SPH}

The Smoothed Particle Hydrodynamics (SPH) method is based on the convolution principle. The approximation $\langle F\rangle$ or smoothed value of a field $F$ at a point $x_{i}$ is given by:

$\left\langle F\left(x_{i}\right)\right\rangle=\int F(x) W\left(\left|x_{i}-x\right|, h\right) d V$

Where $W\left(\left|x_{i}-x\right|, h\right)$ is the kernel function and $h$ is the smoothing length. This kernel function has to have compact support and posess the following properties:

$\int W\left(\left|x_{i}-x\right|, h\right) d V=1$

$W\left(\left|x_{i}-x\right|, h\right) \stackrel{h \rightarrow 0}{\rightarrow} \delta$

If the domain is discretised with a number of particles then the convolution integral is approximated by a sum as follows:

$\left\langle F\left(x_{i}\right)\right\rangle \approx \sum F\left(x_{j}\right) W\left(\left|x_{i}-x_{j}\right|, h\right) \frac{m_{j}}{\rho_{j}}$

Gradients of functions are similarly calculated:

$\left\langle\nabla F\left(x_{i}\right)\right\rangle \approx \sum F\left(x_{j}\right) \nabla W\left(\left|x_{i}-x_{j}\right|, h\right) \frac{m_{j}}{\rho_{j}}$

Applied to hydrocodes these approximations are used to discretise the conservation laws. In the spatial description the conservation of mass, momentum and energy laws are as follows:

$\dot{\rho}=-\rho \nabla \cdot v$ $\rho \ddot{u}=\nabla \cdot \sigma+b$
$\rho \dot{e}=-\sigma: \dot{\varepsilon}$

which results in (Vignjevic et al., 2009):

$$
\begin{aligned}
\left\langle\dot{\rho}_{i}\right\rangle= & -\rho_{i} \sum_{j=1}^{n p} \frac{m_{j}}{\rho_{j}}\left(v_{i}-v_{j}\right) \cdot \nabla_{x_{i}} W\left(\left|x_{i}-x_{j}\right|, h\right) \\
\left\langle\ddot{u}_{i}\right\rangle= & \sum_{j=1}^{n p} \frac{m_{j}}{\rho_{j}}\left(v_{j}-v_{i}\right)\left(v_{R} \cdot \nabla_{x_{i}} W\left(\left|x_{i}-x_{j}\right|, h\right)\right) \\
& -\sum_{j=1}^{n p} m_{j}\left(\frac{\sigma_{i}}{\rho_{i}^{2}}+\frac{\sigma_{j}}{\rho_{j}^{2}}\right) \nabla_{x_{i}} W\left(\left|x_{i}-x_{j}\right|, h\right)+b \\
\left\langle\dot{e}_{i}\right\rangle= & \frac{\sigma_{i}}{\rho_{i}}: \sum_{j=1}^{n p} \frac{m_{j}}{\rho_{j}}\left(v_{i}-v_{j}\right) \nabla_{x_{i}} W\left(\left|x_{i}-x_{j}\right|, h\right)
\end{aligned}
$$

Within the context of a transient dynamics code, the strain rates are required for the constitutive update. They are obtained using the following calculation of the velocity gradient:

$$
\left\langle\nabla v_{i}\right\rangle=\sum_{j=1}^{n p} \frac{m_{j}}{\rho_{j}}\left(v_{j}-v_{i}\right) \nabla W\left(\left|x_{i}-x_{j}\right|, h\right)
$$

It can be seen that the kernel functions which appear in the discretised conservation equations in spatial description are evaluated using the current positions of particles. The kernels evaluated using current particle positions are called Eulerian kernels (Vignjevic et al., 2006; Rabczuk and Belytschko, 2004a).

The equations above are symmetrised (ie. they use differences or sums of variables at particles $i$ and $j$ ). This symmetrisation has the advantage of preserving local conservation of momentum and energy.

Although frequently used the SPH method formulated in this way suffers from tensile instability. The tensile instability was first identified by Swegle et al. (1995) and manifests itself as the development of unphysical fractures or clumping together of particles when the material is subjected to tension. Belytschko and Xiao (2000), Rabczuk and Belytschko (2004a) and Vignjevic et al. (2006) showed that the tensile instability associated with the use of an Eulerian kernel can be avoided by using a Total Lagrangian kernel. Removing tensile instability is crucial in modelling the expanding ring problem as the rings are subjected to large magnitude tensile stresses during the homogeneous expansion phase. The SPH code developed in the Applied Mechanics Department at Cranfield University was used for all simulations. The implementation of the Total Lagrangian formulation in this code is described in (Vignjevic et al., 2006) and will be summarised in the next section. 


\section{Total Lagrangian SPH}

The Total Lagrangian kernel function is evaluated in the initial configuration, in other words the initial coordinates $x_{i}^{0}$ and $x_{j}^{0}$ are used to evaluate the kernel $W$ for given $i$ and $j$ particles.

In the Total Lagrangian SPH formulation the balance equations are written in the initial configuration (material description):

$\rho J=\rho^{0}$

$\rho^{0} \ddot{u}=\nabla_{0} \cdot P+b$

$\rho^{0} \dot{e}=P: \dot{F}$

where $J=|F|$ is the Jacobian of the deformation gradient, $\rho$ density, $u$ displacement, $P$ first Piola Kirchhoff stress, $b$ body force per unit mass, $e$ internal energy. The superscript 0 indicates initial configuration.

The SPH discretisation (Vignjevic et al., 2009, 2006) results in the following equation for the deformation gradient:

$\left\langle F_{i}\right\rangle=-\sum_{j=1}^{n p} \frac{m_{j}}{\rho_{j}^{0}}\left(v_{i}-v_{j}\right) \nabla_{x_{i}^{0}} W\left(\left|x_{i}^{0}-x_{j}^{0}\right|, h^{0}\right)$

From this equation the conservation of mass can be used to calculate the current density:

$\rho=J^{-1} \rho^{0}$

The conservation of momentum equation becomes:

$\left\langle\ddot{u}_{i}\right\rangle=-\sum_{j=1}^{n p} m_{j}\left(\frac{P_{i}}{\rho_{i}^{02}}+\frac{P_{j}}{\rho_{j}^{02}}\right) \nabla_{x_{i}^{0}} W\left(\left|x_{i}^{0}-x_{j}^{0}\right|, h^{0}\right)+b$

The energy update is calculated as follows:

$\left\langle\dot{e}_{i}\right\rangle=\frac{P_{i}}{\rho_{i}}: \sum_{j=1}^{n p} \frac{m_{j}}{\rho_{j}^{0}}\left(v_{i}-v_{j}\right) \nabla_{x_{i}^{0}} W\left(\left|x_{i}^{0}-x_{j}^{0}\right|, h^{0}\right)$

The constitutive update in Cranfield University's SPH code uses a rate of deformation tensor $\dot{\varepsilon}$ to calculate a stress increment. The rate of deformation tensor can be obtained from the deformation gradient using the Green-Lagrange strain rate tensor $\dot{E}$ :

$\dot{E}=\frac{1}{2}\left(\dot{F}^{T} F+F^{T} \dot{F}\right)$

and the following expression:

$\dot{\varepsilon}=F^{-T} \dot{E} F^{-1}$

The Cauchy stress $\sigma$ obtained from the constitutive update is transformed to the 1st Piola-Kirchhoff stress $P$ for use in the conservation of momentum equation using:

$P=J F^{-1} \sigma$
A cubic B-spline kernel function was used. The kernel functions were normalised (Shepard functions) to restore zero order consistency:

$W\left(\left|x_{i}^{0}-x_{j}^{0}\right|, h^{0}\right)=\frac{\tilde{W}\left(\left|x_{i}^{0}-x_{j}^{0}\right|, h^{0}\right)}{\sum_{j=1}^{n p} \frac{m_{j}}{\rho_{j}^{0}} \tilde{W}\left(\left|x_{i}^{0}-x_{j}^{0}\right|, h^{0}\right)}$

Where $\tilde{W}$ is the standard cubic B-spline kernel. These semi-discretised equations were integrated in time using a central difference integration scheme (explicit time integration). The update of particle positions was performed using a smoothed velocity (XSPH) (Randles and Libersky, 1996). The motivation for using the SPH method is that due to its meshless nature the $\mathrm{SPH}$ method is less directionally sensitive compared to a finite element mesh which has preferential directions for cracks growth or localisation. Furthermore the smoothing properties of the kernel interpolation in the $\mathrm{SPH}$ method may make it less sensitive to deformation localisation related to material softening.

\subsection{Constitutive Behaviour}

The constitutive model is based on an additive decomposition of the strain rate tensor. Elastic behaviour was assumed to be isotropic and is defined through the generalised Hooke's law. Plasticity is described using a J2 flow theory with isotropic hardening. The modified Johnson Cook yield function and an associative flow rule are used. The Jaumann stress rate is used to update (the stress tensor and a radial return algorithm is used for the integration of the constitutive equations. The volumetric part of deformation is described by the MieGruneisen equation of state. The Johnson-Cook material model is routinely used for problems where high strain rates and temperatures occur. Similarly to Randles and Libersky (1996) a modification to the JohnsonCook model was made because the original JohnsonCook model is limited to strain rates up to $10^{3} \mathrm{~s}^{-1}$. The modification used in this work is by adding an extra term:

$\left(\frac{\dot{\bar{\varepsilon}}}{\bar{d}}\right)^{E}$

This term is identical to the term of the CowperSymonds strain rate dependent model as shown in Equation 25.

$$
\begin{aligned}
\sigma_{Y}= & \left(A+B \bar{\varepsilon}_{p l}^{n}\right) \\
& \left(1+C \ln \left(\frac{\dot{\bar{\varepsilon}}}{\overline{\bar{\varepsilon}}}\right)+\left(\frac{\dot{\bar{\varepsilon}}}{d}\right)^{E}\right)\left(1-T^{* m}\right)
\end{aligned}
$$

In addition to the Johnson-Cook model the MieGruneisen equation of state was used (LSTC, 2007). 
Table 1 Parameters for Johnson-Cook model used for aluminium 6061-O

\begin{tabular}{ll}
\hline Property & Value \\
\hline$G$ & $77.0 \mathrm{GPa}$ \\
$A$ & $25.0 \mathrm{MPa}$ \\
$B$ & $185 \mathrm{MPa}$ \\
$n$ & 0.28 \\
$C$ & 0.002 \\
$d$ & 0.08 \\
$E$ & 0.5 \\
$m$ & 0.75 \\
$T_{m}$ & $925.0 \mathrm{~K}$ \\
$T_{r}$ & $293.0 \mathrm{~K}$ \\
$\dot{\epsilon}_{0}$ & $1.0 \mathrm{~s}^{-1}$ \\
$\Gamma$ & 1.97 \\
$c$ & $5240 \mathrm{~m} / \mathrm{s}$ \\
$S_{1}$ & 1.4 \\
$S_{2}$ & 0.0 \\
\hline
\end{tabular}

The values for $\Gamma, c, S_{1}, S_{2}$ are those for aluminium 6061-T6 and were taken from Corbett (2006) and summarised in Table 1. The values for the other material model parameters were mainly based on the data provided in Zhang and Ravi-Chandar (2006). The data is summarised in Table 1. The value of $c$ is taken from Corbett (2006). $G$ is the shear modulus of the material.

\subsection{Damage Model}

A combination of the Johnson-Cook failure model with a ductile damage model developed by Lemaitre was used in this work. The phenomenological Lemaitre (Lemaitre $\tilde{e}_{Y}=\frac{\sigma_{Y}}{1-D}$ and Sermage, 1997) damage model is based on thermodynamics of irreversible processes. It can be used to model brittle and ductile isotropic damage. For ductile damage strain to fracture strongly decreases when triaxiality ratio increases. The Lemaitre damage model describes the damage growth rate $\dot{D}$ as a function of the effective plastic strain rate $\dot{\bar{\varepsilon}}_{p l}$ as follows (more details can be found in Lemaitre and Chaboche (2007); Lemaitre and Sermage (1997)):

$\dot{D}=\left(-\frac{Y}{S}\right)^{t} \dot{\bar{\varepsilon}}_{p l} \quad$ if $\quad \bar{\varepsilon}_{p l} \geq \bar{\varepsilon}_{\text {threshold }}$

$\dot{D}=0 \quad$ if $\bar{\varepsilon}_{\text {pl }}<\bar{\varepsilon}_{\text {threshold }}$

with

$-Y=\frac{\sigma_{e q}^{2}}{2 E(1-D)^{2}}\left(\frac{2}{3}(1+\nu)+3(1-2 \nu)\left(\frac{-p}{\sigma_{e q}}\right)^{2}\right)$

$\sigma_{e q}$ is the equivalent stress (von Mises stress), $\nu$ is Poisson's ratio, $E$ Young's modulus and $p$ pressure. Damage $D$ starts growing when a threshold effective plastic
Table 2 Parameters for Lemaitre damage model used for aluminium 6061-O

\begin{tabular}{ll}
\hline Property & Value \\
\hline$S$ & $0.625 \mathrm{MPa}$ \\
$t$ & 1.0 \\
$D_{c}$ & 0.5 \\
$\bar{\varepsilon}_{\text {threshold }}$ & 0.15 \\
$\alpha$ & 10.0 \\
$\beta$ & 0.25 \\
\hline
\end{tabular}

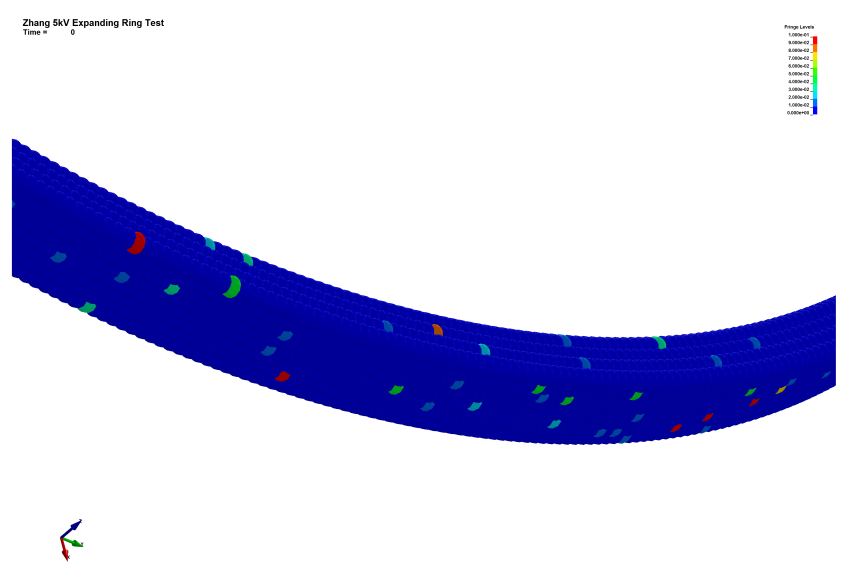

Fig. 2 Initial Damage in Expanding Ring Model

strain is reached (see Equation 26), and continues until a critical damage level $D_{c}$ is reached. At that point fracture occurs and the deviatoric stresses for the SPH particle under consideration are set to zero. With the evolution of damage the yield stress $\sigma_{Y}$ and shear modulus $G$ are degraded as follows:

$\tilde{G}=\frac{G}{1-D}$

The parameters used in this work are summarised in Table 2. When failure occurs the stress in the particle is set to zero and is removed from the neighbour lists thus ensuring that the particle no longer interacts directly with neighbour particles. The initial level of damage was randomised using a Weibull distribution with a mean value of 0.0024 and standard deviation of 0.00397 for the initial damage (see Figure 2). A similar approach was recently used by Meyer et al. (2012) and Hopson et al. (2011) to model statistical variations in 27) fracture properties for ballistic impact and explosively loaded cylinders problems respectively. Since it can be assumed that the rings used in the electromagnetically driven ring experiments were free of macroscopic defects, the level of initial damage should be small. Since 
the critical level of damage was 0.5 a mean initial damage level of 0.0024 appears reasonable. The results are sensitive to the Weibull distribution parameters, which is in agreement with the observations of Meyer et al. (2012) and Hopson et al. (2011). Other values for the Weibull distribution parameters would either result in too few - or too many fragments, or in fracture at a time different from the experiments. The lower expansion speed models were more sensitive to these changes in the initial damage distribution. The damage distribution is also dependent on the discretisation density. For a different discretisation density an equivalent damage distribution can be calculated using the approach described Meyer et al. (2012) and Hopson et al. (2011).

\subsection{Treatment of Failure and Fracture}

To allow fractures to develop realistically in SPH it is not sufficient to simply set the stress of a failed particle to zero and to remove it from the neighbour lists. As shown in Figure 3 if we consider a particle $i$ which has a neighbour particle $j_{1}$ which has failed, then other neighbour particles, like particle $j_{2}$, which are obscured by particle $j_{1}$ should also be removed from the neighbour list of particle $i$. If this is not done the stress states at particle $i$ are unphysical. If an Eulerian kernel is used then the interaction between the particles $i$ and $j_{2}$ which are separated by a crack would gradually reduce as they move apart. Although not physically correct a free surface would form when the particles move out of the kernel's support (ie. stop interacting). However, if a Total Lagrangian SPH formulation is used then the neighbour lists are fixed and kernels and kernel derivatives are evaluated for the initial particle locations only. Therefore it is necessary to explicitly deal with particles that are obscured by a crack (failed particles). In this study, a particle visibility was considered by introducing a truncated cone as shown in Figure 4. The top of the truncated cone is located on the failed particle. The diameter of top of the truncated cone is equal to the initial particle spacing. The angle of the cone is such that the tip of the equivalent cone is located at the $\mathrm{i}$ particle (see Figure 4). If a particle lies inside this cone it removed from the neighbour list of the particle $i$.

\subsection{Electromagnetic Forces}

In the SPH model, the electromagnetic loading was applied as a body force defined by the 1D analytical expression for the ring acceleration as a function of ring current proposed by Zhang and Ravi-Chandar (Zhang
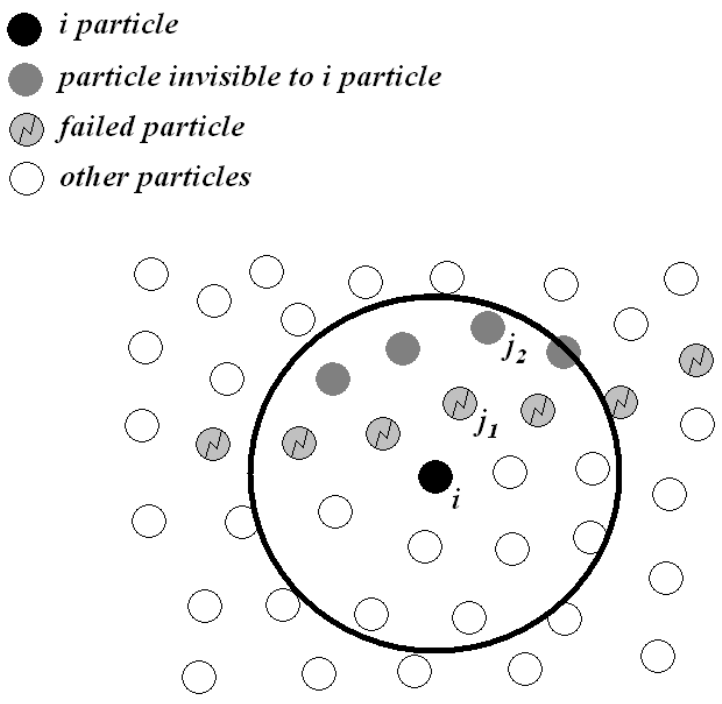

Fig. 3 Different types of neighbour particles in the presence of failed particles

i particle
particle invisible to i particle
(1) failed particle
other particles

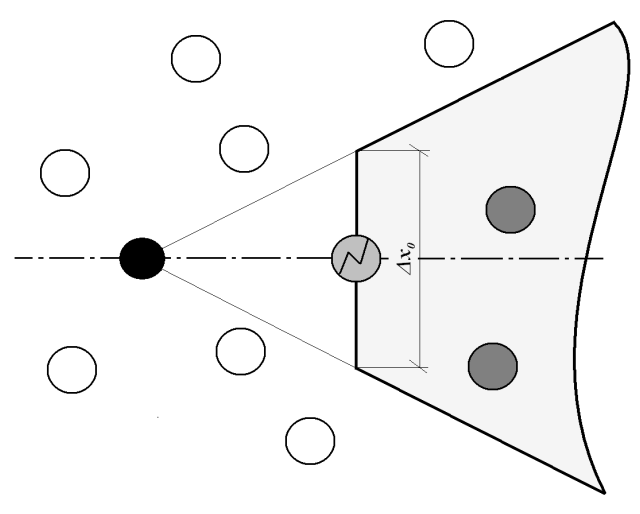

Fig. 4 Visiblity criterion based on truncated cone (shaded area indicates truncated cone)

and Ravi-Chandar, 2006). The expression they used is given in Equation 30.

$\ddot{r}=\frac{I_{1} I_{2}}{2 \pi r A \rho} \frac{d L_{12}}{d r}+\frac{I_{2}^{2}}{4 \pi r A \rho} \frac{d L_{22}}{d r}-\frac{\sigma}{\rho r}$

This means that the loading has been applied by using the measured currents in the ring and solenoid as input data. The effect of the resulting electromagnetic forces was incorporated by adding a body force equivalent to the first two terms in Equation 30 to the right hand 
side of the momentum Equation 18. The terms $L_{22}$ and $L_{21}$ are function of the radius as defined in Equations 31 and 33 and hence need to be recalculated at every timestep.

$L_{22} \approx 4 \pi r\left[\ln \frac{8 r}{r_{w}}-\frac{7}{4}\right] 10^{-7}$

where

$r_{w}=\sqrt{\frac{A}{\pi}}$

$\frac{1}{2 \pi r} \frac{d L_{12}}{d r}=\frac{\mu_{0}}{2 \pi} \sum_{i=0}^{n} \int_{0}^{\pi} \frac{r_{i}^{2}-r_{i} r \cos \theta}{\left(r^{2}-2 r r_{i} \cos \theta+r_{i}^{2}+z_{i}^{2}\right)^{\frac{3}{2}}} d$

Since these terms depend on the radius of the ring it is clear that the electro-magnetical and thermo-mechanical models are coupled.

\subsection{Effect of Joule Heating}

The effect of large currents on temperature can not be ignored. Zhang and Ravi-Chandar (2006) calculated the temperature increase, and obtain temperature increases ranging from $250^{\circ} \mathrm{C}$ to almost $500^{\circ} \mathrm{C}$. This increase in temperature will have a significant effect on the constitutive behaviour of the material. A similar observation has also been made by Meyers (1994). The rate of increase of temperature as a function of the ring current and plastic work are defined as follows in Zhang and Ravi-Chandar (2006).

$\dot{T}=\frac{R_{2} I_{2}^{2}}{2 \pi r A C_{p} \rho}+\frac{\sigma \dot{\epsilon}_{p}}{C_{p} \rho}$

Only the first term in Equation 34 related to the Joule heating was added to the temperature calculation for each particle. Including the temperature increase due to Joule heating in this way implies that it was assumed that the current density is homogeneous in the cross section and the cross sectional area is independent of angular position. This last assumption is only valid up to the point at which necking occurs. In the necked region the current density would increase and this would lead to higher temperatures in the neck. However, accurately dealing with this effect would require fully coupled 3D electromagnetic and thermomechanical models. Due to the uncertainty in constitutive data, the data is taken from the literature, and the complexity of modelling this effect it was not taken into account in the models.

The second term in equation 34 related to temperature increase due to plastic work is accounted for in the constitutive equation of the SPH solver using the following equation:

$\dot{T}=\frac{\beta \sigma: \dot{\epsilon}_{p}}{C_{p} \rho}$

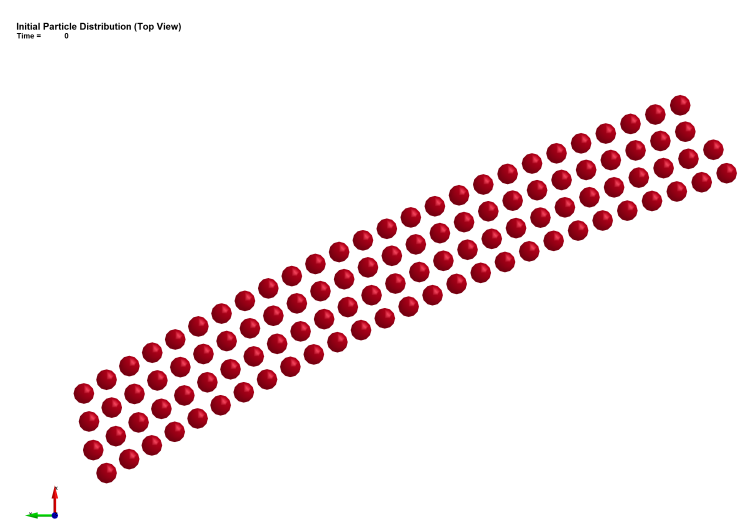

Fig. 5 Initial Positioning of Particles

This equation is the multi-axial equivalent of the second term in equation 34 and also includes the TaylorQuinney coefficient $\beta$ which defines the fraction of plastic work which is converted into heat. A value for $\beta$ of 0.9 has been used in all simulations.

\section{Description of Models}

\subsection{Particle Positioning}

The initial configuration of the model is shown in Figure 2. As explained above the input loading was defined in terms of the currents measured in the primary circuit (capacitor-switch-coil) and the current measured in the ring. Although fracture occurs between $30 \mu \mathrm{s}$ and $70 \mu \mathrm{s}$, the simulations were run until $120 \mu \mathrm{s}$. This was done in order to allow for completion of the necking processes and elastic unloading of all the fragments. To avoid any preferential fracture position or direction the particles in the ring were distributed in a particluar manner. The particles were positioned on a series of planes perpendicular to the axis of the ring. The distance between these planes was the integer value of the ring height divided by the desired particle spacing. Within each of these planes the particles were located on concentric circles as shown in Figure 5. The radial distance between these circles was again equal to the integer value of the ring thickness divided desired particle spacing. The individual particles were then distributed along these circles (See Figure 5), again the number of particles on each circle was the integer value of the circle length divied by the disired particle spacing. The position of the first particle on each ring was at a random point on the circle to avoid preferential cross sectional planes along which a crack can form. A second advantage of this approach is that all particles have the same mass. 


\subsection{Discretisation Density}

In order to determine which discretisation is able to capture localisation a sensitivity study has been performed on a bar subjected to a constant strain rate. This setup introduces similar loading to an expanding ring experiment. A similar setup was used in the Finite Element models in Zhang and Ravi-Chandar (2006) and Zhang and Ravi-Chandar (2008). The constant strain rate was achieved by fixing one end of the bar, defining a prescribed velocity $v_{\text {end }}$ to the other end and initialising the velocity field as linearly varying from 0 to $v_{\text {end }}$. The bar had the same cross section as the rings $(0.5$ by $1.0 \mathrm{~mm}$ ) and length of $1.9164 \mathrm{~mm}$. In order to study localisation a defect was introduced halfway along the bar. Similarly to Zhang and Ravi-Chandar (2006) the defect was defined in the form of an area ( 0.250 by $0.250 \mathrm{~mm}$ ) which does not exhibit any strain hardening. This defect will cause a neck to form. The neck then develops into a complete fracture of the bar and release waves unload the rest of the bar. Three different particle spacings $0.0125 \mathrm{~mm}, 0.00625 \mathrm{~mm}$ and $0.003125 \mathrm{~mm}$ were used, resulting in models of rerspectively 5664, 44800 and 365864 particles. The results are shown in figures 6 and 7 . Figure 6 shows that shape of the fractured necks for the three particle spacings is similar. The evolution of the effective plastic strain for two points is shown in Figure 7. One point is located in the neck while the other point is located outside the neck. It can be seen that inside the neck the plastic strain increases rapidly during necking. The plastic strain in the point outside the neck increases linearly until that point is unloaded. This unloading occurs at a time of $25.0 \mu \mathrm{s}$ for the three particle spacings, and the plastic strain is around 0.22 , the Considère strain for this material. Based on these results it was concluded that having 4 particles in the radial and 8 in the axial direction was sufficient to capture localisation. The total number of particles in the expaning ring models was 24904 .

\subsection{Influence of Modelling Assumptions}

In order to assess the influence of the various modelling assumptions six hierarchically different models were developed and used for simulation of a range of the expanding ring experiments. The simplest model was Model 1 and the most complex model was Model 6 . The results for the lowest ( $5 \mathrm{kV}$ charge) and highest (8kV charge) expansion speeds are summerised in Table 3. In the baseline Model 1 the standard Johnson-Cook model (i.e. model without the additional strain rate dependent term 24 was used and damage and the effect of plastic work on temperature were neglected. The effect
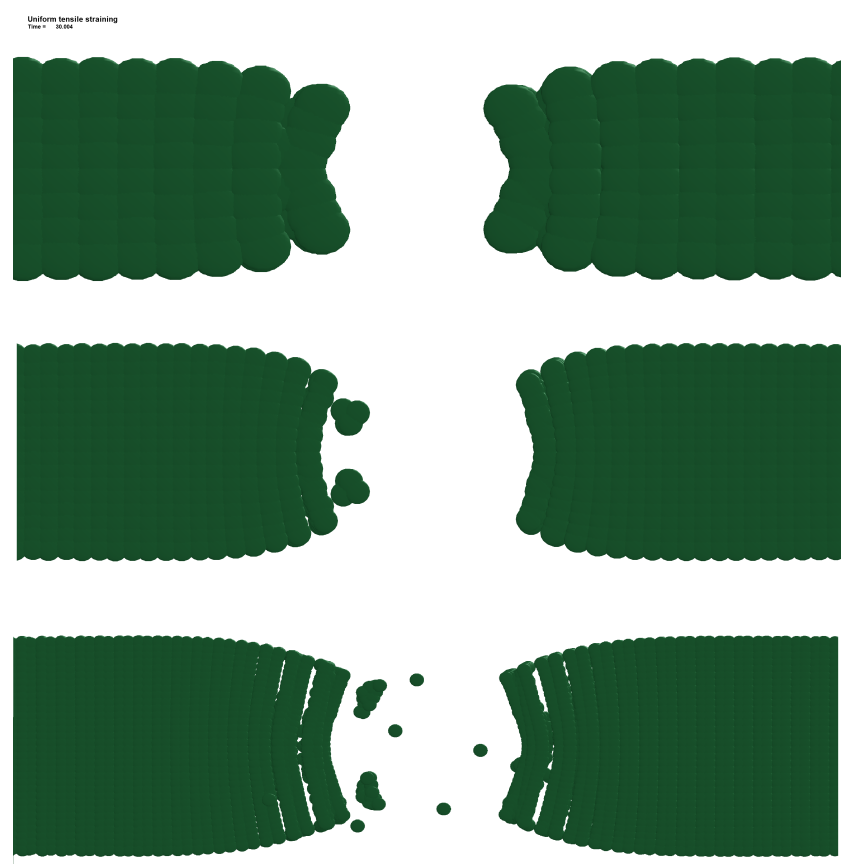

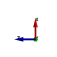

Fig. 6 Necks in bar subjected to constant strain rate

of Joule heating was taken into account. As expected in the absence of damage in the model no fragmentation occured, however a number of necks do develop during the ring expansion. The damage model was included in Model 2. However, the inital damage was not randomised using a weibull distrubtion but set to 0 for all particles. Although fragmentation occured, the number of fragments was significantly overestimated and the time at which the first fracture occured was underestimated. The modification to the strain rate term in the constitutive model was added in Model 3. This model underestimated the number of fragments and the error in the time to the first fracture increased. The effect of plastic work on temperature was included in Model 4. This had a small influence on the fracture time. Model 5 was generated by randomising the intitial damage in Model 2. In this case fragment numbers for the higher expansion speed drop significantly. Similarly the time to fracture was more than halved. Model 6 combined the previous modifications and predicts the number of fragments and the time at which the first fracture occurs quite well. This demonstrates that all relevant physics needs to be taken into account to obtain realistic results over a range of test conditions. The importance of accurate constitutive and damage models is also identified by Guduru and Freund (2002) and Zhang and Ravi-Chandar (2009b). 

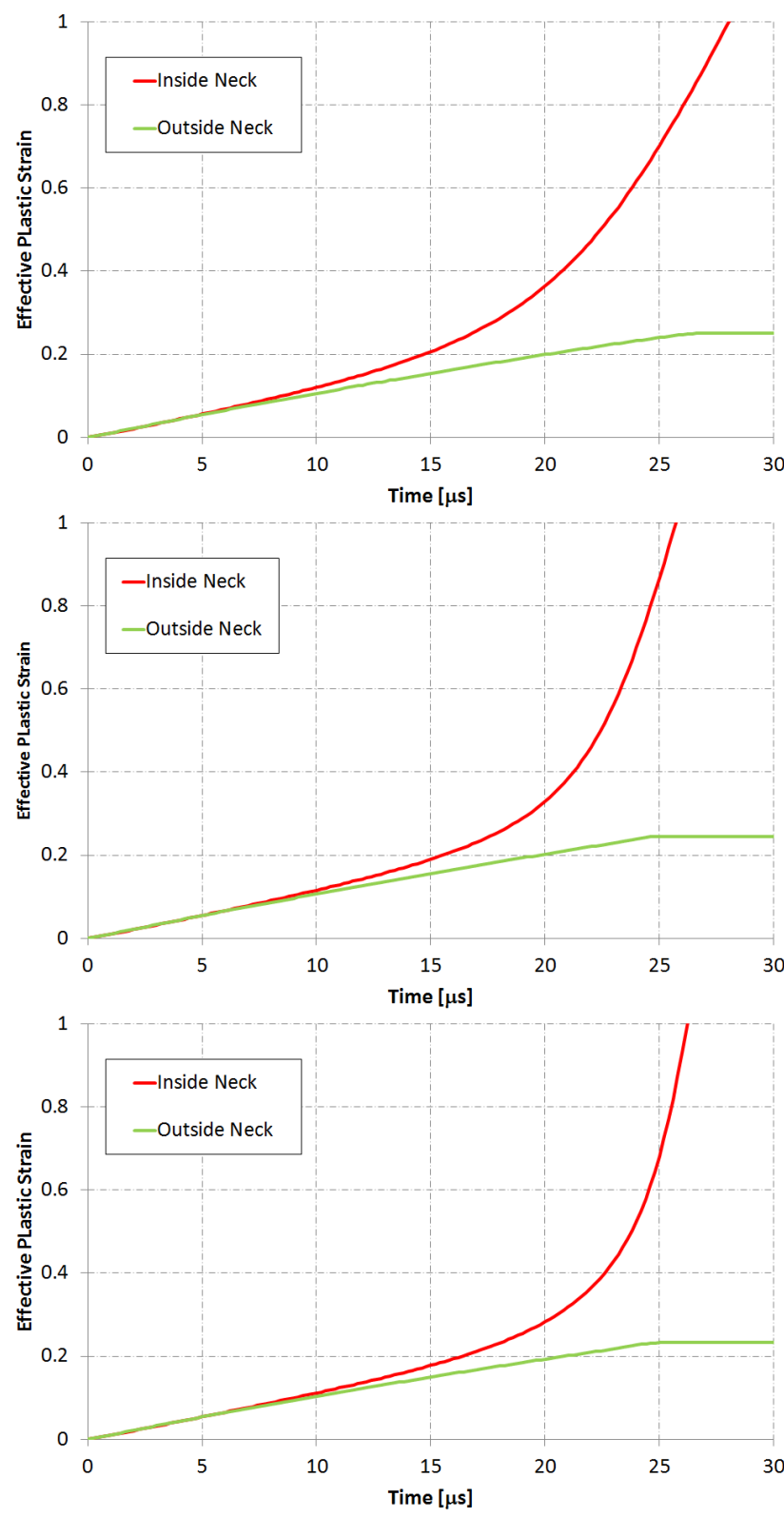

Fig. 7 Evolution of effective plastic strain in and outside the necked region in bars

\section{Results and Discussion}

Using these model parameters the four experiments $(5 \mathrm{kV}$, $6 \mathrm{kV}, 7 \mathrm{kV}$ and $8 \mathrm{kV}$ charge) were simulated. The final state (after $120 \mu \mathrm{s}$ ) of these four simulations is shown in Figures 8 to 15. These figures show the level of damage in the rings on a scale of 0.0 (no damage) to 0.5 (= critical damage $D_{c}$ i.e. failure).
Table 3 Effect of Model Assumptions on Fragmentation Behaviour

\begin{tabular}{|c|c|c|c|c|}
\hline \multirow[t]{2}{*}{ Assumptions } & \multicolumn{2}{|c|}{$5 k V$ Experiment } & \multicolumn{2}{|c|}{$8 k V$ Experiment } \\
\hline & $\begin{array}{l}\text { Number } \\
\text { of } \\
\text { Frag- } \\
\text { ments }\end{array}$ & $\begin{array}{l}\text { Time } \\
\text { of } 1^{\text {st }} \\
\text { Frac- } \\
\text { ture }\end{array}$ & $\begin{array}{l}\text { Number } \\
\text { of } \\
\text { Frag- } \\
\text { ments }\end{array}$ & $\begin{array}{l}\text { Time } \\
\text { of } 1^{\text {st }} \\
\text { Frac- } \\
\text { ture }\end{array}$ \\
\hline $\begin{array}{l}\text { Model } 1 \text { (Baseline } \\
\text { Model): No damage, } \\
\text { No strain rate mod- } \\
\text { ification, No heating } \\
\text { due to plastic work, } \\
\text { No randomisation }\end{array}$ & 0 & $\mathrm{~N} / \mathrm{A}$ & 0 & $\mathrm{~N} / \mathrm{A}$ \\
\hline $\begin{array}{l}\text { Model 2: No strain } \\
\text { rate modification, } \\
\text { No heating due to } \\
\text { plastic work, No } \\
\text { randomisation }\end{array}$ & 10 & $94 \mu s$ & 26 & $78 \mu s$ \\
\hline $\begin{array}{l}\text { Model 3: No heating } \\
\text { due to plastic work, } \\
\text { No randomisation }\end{array}$ & 0 & $\mathrm{~N} / \mathrm{A}$ & 3 & $106 \mu s$ \\
\hline $\begin{array}{l}\text { Model 4: No ran- } \\
\text { domisation }\end{array}$ & 0 & $\mathrm{~N} / \mathrm{A}$ & 3 & $112 \mu s$ \\
\hline $\begin{array}{l}\text { Model 5: No strain } \\
\text { rate modification, } \\
\text { No heating due to } \\
\text { plastic work }\end{array}$ & 12 & $38 \mu \mathrm{s}$ & 14 & $30 \mu s$ \\
\hline $\begin{array}{l}\text { Model } 6 \text { (Final } \\
\text { Model): with dam- } \\
\text { age, strain rate } \\
\text { modification, heat- } \\
\text { ing due to plastic } \\
\text { work and randomi- } \\
\text { sation }\end{array}$ & 4 & $58 \mu \mathrm{s}$ & 12 & $42 \mu s$ \\
\hline
\end{tabular}

\subsection{Number of Fragments and Necking}

The number of fragments in simulations and experiments is summarised in Table 4 and Figure 16 for all tests. For the $5 \mathrm{kV}$ experiment it can be seen in Figures 8 and 9 that the simulation predicts 4 fragments, which compares well with the 3 fragments observed in the test. On top of complete fractures a number of arrested necks can be identified. Some necks are well developed and clearly show a reduction in cross section. Other necks are less developed but can be identified by a localised increase in damage. The width of the necks is roughly equal to the ring height which is what was observed in the experiments (Zhang and Ravi-Chandar, 2006). The simulation results for the $6 \mathrm{kV}$ experiment (Figure 10 and 11) are similar. In accordance with experiments the number of fragments increases. The simulation predicts 5 fragments while the 2 tests performed resulted in 6 and 8 fragments respectively. A number of arrested necks can be observed. The simulation of the $7 \mathrm{kV}$ ex- 


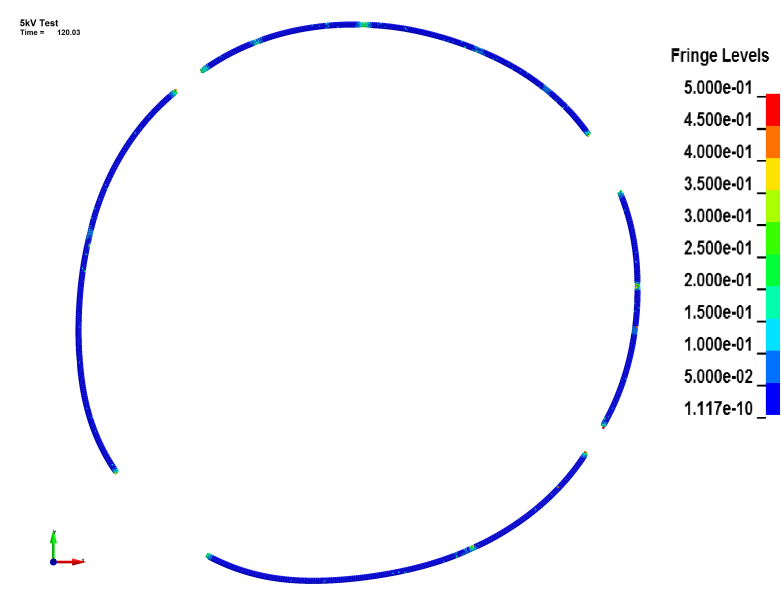

Fig. 8 Fragments and Damage Distribution in $5 \mathrm{kv}$ model after $120 \mu \mathrm{s}$

periment (Figure 12 and 13) results in 8 fragments (10 fragments in the test). Apart from arrested necks, in Figure 12 one can also observe a number of fragments which show some bending around the necked area. This is consistent with the test results in (Zhang and RaviChandar, 2006). Finally, the simulation results for the $8 \mathrm{kV}$ experiment (Figure 14 and 15) result in 12 fragments (12 fragments in the test). Again arrested necks and fragments which bend around a necked area can be observed in Figure 14. It can be seen that as the expansion speed increases the number of fragments increases. In Figure 16 the number of fragments in the simulations and experiments is plot as a function of radial velocity of the ring at a time $t=40 \mu \mathrm{s}$. Since the radial velocity is dependent on time a choice has to be made on a nominal expansion speed for each experiment. In a first phase the velocity will increase due to the electromagnetic force. As the currents in the ring drop the driving force will gradually drop to zero and from then onwards the ring will expand freely. During this stage the velocity drops as kinetic energy in converted into strain energy. The nominal expanding velocity in the tests is the velocity at the start of free expansion stage, which occurs after roughly $40 \mu \mathrm{s}$. Hence, the radial velocity in the simulations has been taken as the expansion velocity of the ring at $40 \mu \mathrm{s}$. It can be seen that the simulation results follow the same trend as the experimental data. Taking into account the scatter in experimental data, the complexity of the model and the fact many constitutive and damage parameters are taken from the literature the correlation with the experimental data is good. The RMS error between simulation and test results for the number of fragments is 1.8 fragments.

In order to check the plastic strain in the fragments the evolution of plastic strain at a number of points was plot as a function of time, similarly to section 5.2.

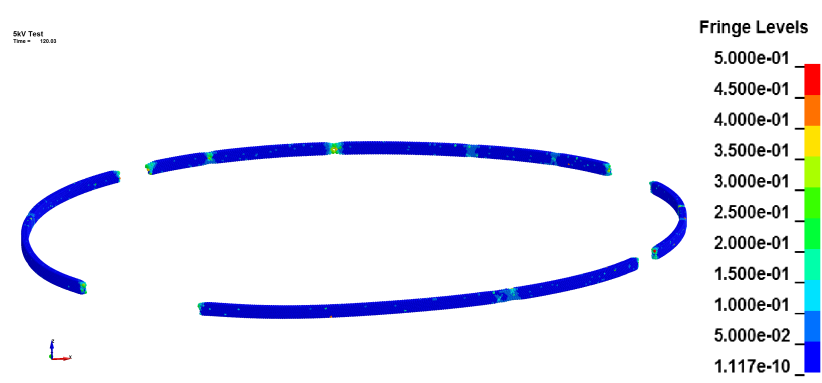

Fig. 9 Arrested Necks in 5kv model after $120 \mu \mathrm{s}$

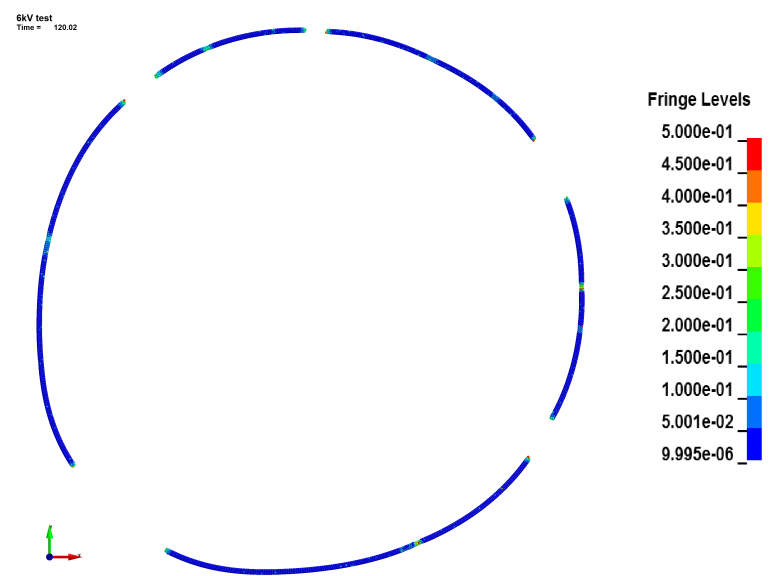

Fig. 10 Fragments and Damage Distribution in 6kv model after $120 \mu \mathrm{s}$

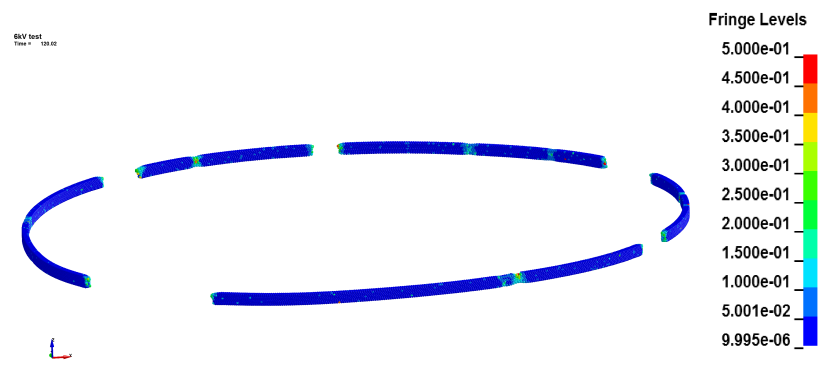

Fig. 11 Arrested Necks in $6 \mathrm{kv}$ model after $120 \mu \mathrm{s}$ 


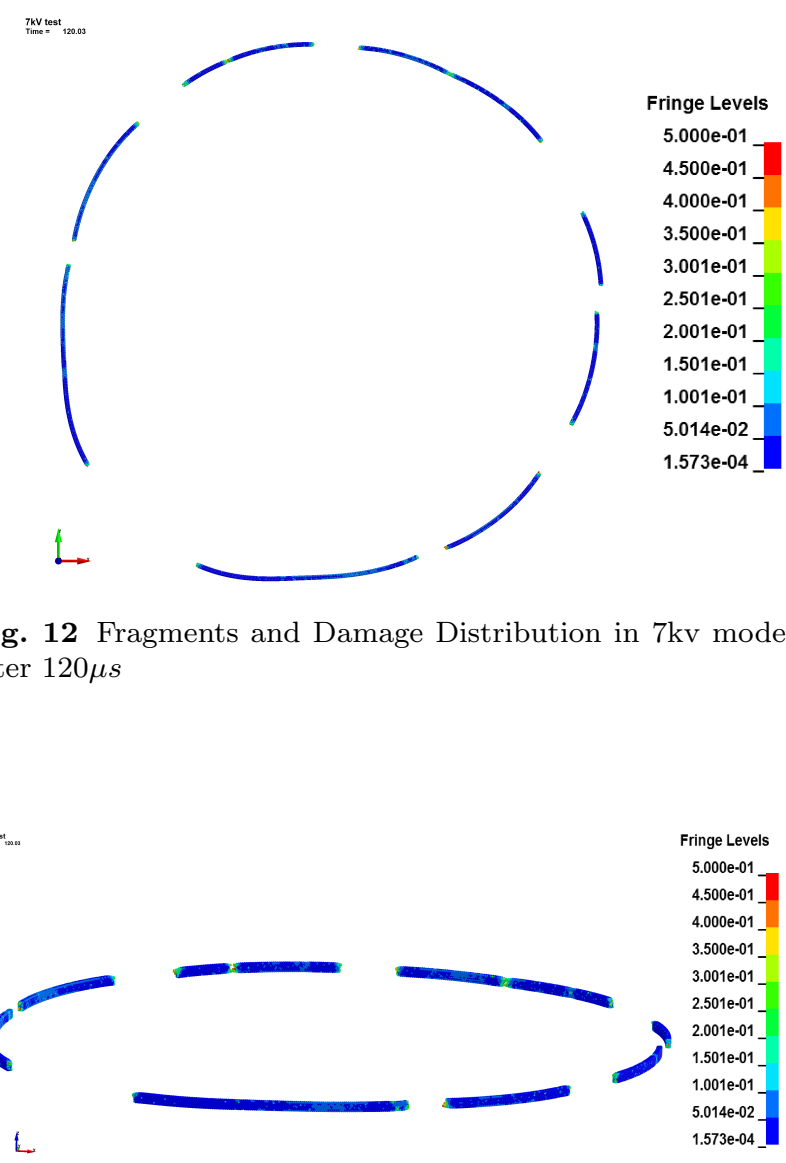

Fig. 15 Arrested Necks in $8 \mathrm{kv}$ model after $120 \mu \mathrm{s}$

Table 4 Comparison of Measured and Simulated Number of Fragments

\begin{tabular}{|c|c|c|}
\hline \multirow[t]{2}{*}{ Test } & \multicolumn{2}{|c|}{ Number of Fragments } \\
\hline & Experiment & Simulation \\
\hline $5 k V$ & 3 & 4 \\
\hline $6 k V$ & 7 & 5 \\
\hline $7 k V$ & 10 & 8 \\
\hline $8 k V$ & 12 & 12 \\
\hline
\end{tabular}

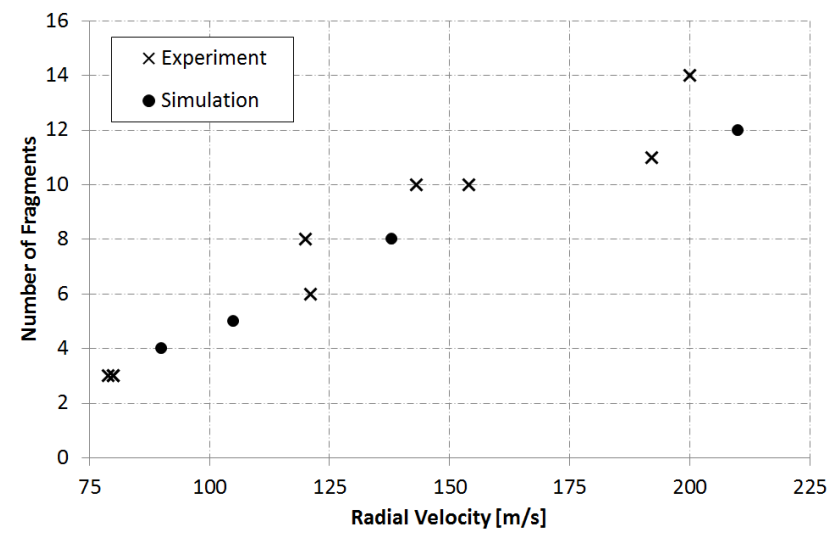

Fig. 16 Comparison of Measured and Simulated Number of Fragments as function of Radial Expansion Velocity

The results for the $5 \mathrm{kV}$ and $8 \mathrm{kV}$ tests are shown in figures 17 and 18. In both cases three points have been plot: a point in a neck, a point near the neck which first develops into a fracture, and a point diametrically opposite to this neck. It can be seen that in the neck the effective plastic strain increases rapidly during necking (red curve) and that near the first neck the strain is limited to a value close to the Considère strain (green curve). However fragments further away from the first fracture tend to show a higher strain (blue curve in the graphs), especially in the experiment at higher expan- 


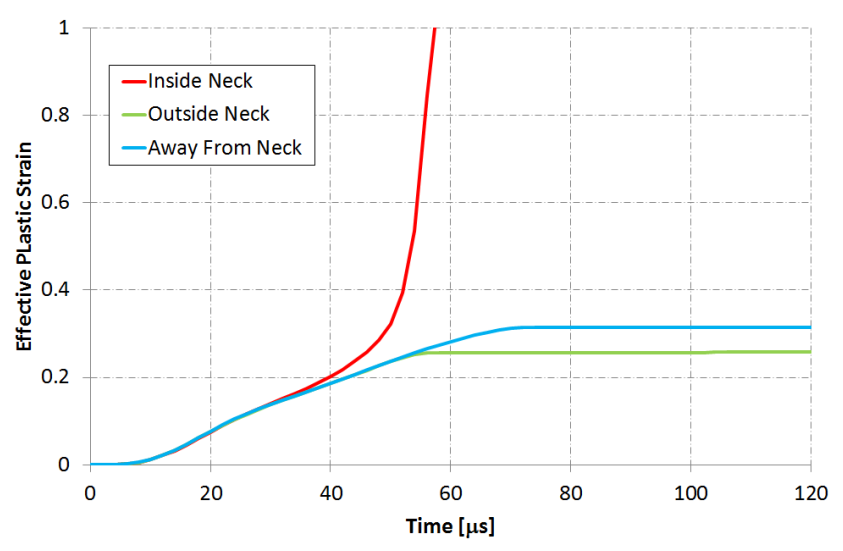

Fig. 17 Evolution of effective plastic strain versus time in and outside the necked region for the $5 \mathrm{kV}$ experiment

sion speed (figure 18). This can be explained by the fact that points further away from a fracture will continue to accumulate plastic deformation until a Mott release wave unloads them. This would then indicate that the development of local defects into a neck and subsequent fracture across the whole specimen cross section does not happen fast enough throughout the whole ring. In order to explain this difference it is useful summarise the findings of Zhang and Ravi-Chandar (2008). In this paper these authors discuss the effect of absolute cross section size on necking and fragmentation. They consider that localisation of deformation occurs in a small volume and that this localisation then has to propagate across the whole cross section to develop into a neck. In expanding ring tests of $\mathrm{Al}$ 6061-O specimen with larger cross sections they observe that the onset of necking occurs at strains of 0.45 , in other words higher than the Considère strain of 0.22 . They confirm this with finite element model results of samples with different cross section size. Therefore they conclude that localisation strain depends on the absolute size of the specimen and that this is due inertial effects on the propagation of the localisation across the specimen width. Based on this, a possible explanation for the higher plastic deformations away from the first necks may be that although localisation starts at the right time some localisations do not develop into necks fast enough. This may be due to inaccuracies in the constitutive and damage model and/or the randomisation of initial damage. Since the constitutive model has been used with data obtained from the literature it would be necessary to characterise the material for the range of strains, strain-rates and temperatures experienced during the tests before investigating this further. This characterisation should cover elasto-plastic behaviour as well as damage evolution.

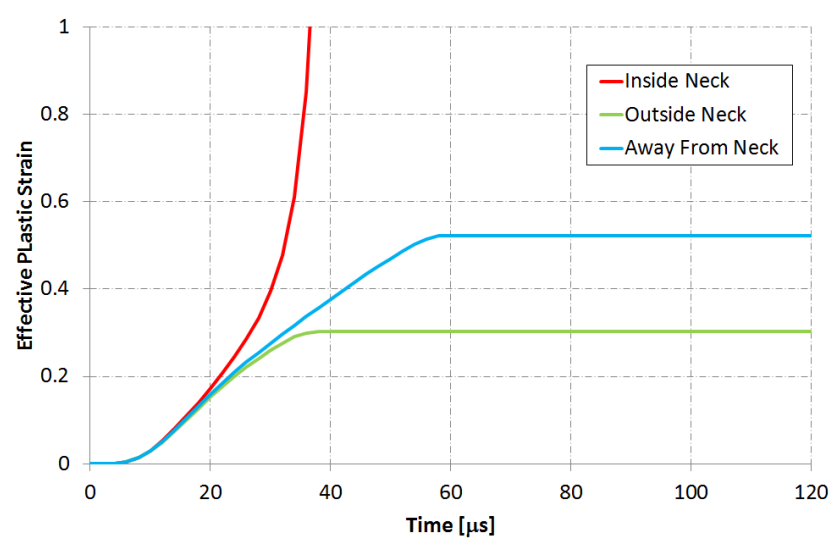

Fig. 18 Evolution of effective plastic strain versus time in and outside the necked region for the $8 \mathrm{kV}$ experiment

Table 5 Comparison of Measured and Simulated Time to First Fracture

\begin{tabular}{lll}
\hline \multirow{2}{*}{ Test } & \multicolumn{2}{l}{ Time of First Fracture } \\
& Experiment & Simulation \\
\hline $5 k V$ & $57.7 \mu \mathrm{s}$ & $58.0 \mu \mathrm{s}$ \\
$6 k V$ & $47.99 \mu \mathrm{s}$ & $56.0 \mu \mathrm{s}$ \\
$7 k \mathrm{~V}$ & $38.7 \mu \mathrm{s}$ & $44.0 \mu \mathrm{s}$ \\
$8 k \mathrm{~V}$ & $40.55 \mu \mathrm{s}$ & $42.0 \mu \mathrm{s}$ \\
\hline
\end{tabular}

\subsection{Time of First Fracture}

The time at which the first fracture occurs in simulations and experiments is summarised in table 5 and Figure 19 for all tests. For the $5 \mathrm{kV}$ experiment it can be seen that the simulation predicts that after $57.7 \mu \mathrm{s}$ the first fracture occurs. This compares well to the test result of $58.0 \mu \mathrm{s}$. The simulation results for the $6 \mathrm{kV}$ experiment shows that compared to the $5 \mathrm{kV}$ the time to first fracture in the simulation is reduced to $56.0 \mu \mathrm{s}$, in the test this was $47.99 \mu \mathrm{s}$. The simulation of the $7 \mathrm{kV}$ experiment results in a further reduction in time to first fracture to $44.0 \mu \mathrm{s}(38.7 \mu \mathrm{s}$ in the test). Finally, in the simulation results for the $8 \mathrm{kV}$ experiment a time to first fracture to $42.0 \mu \mathrm{s}$ is obtained $(40.55 \mu \mathrm{s}$ in the test). It can be seen that as the expansion speed increases the time at which the first fracture occurs decreases. The simulation results follow the same trend as the experimental data. The correlation with the experimental data is good and the RMS error between simulation and test results for the time at which the first fracture occurs is $4.9 \mu \mathrm{s}$. 


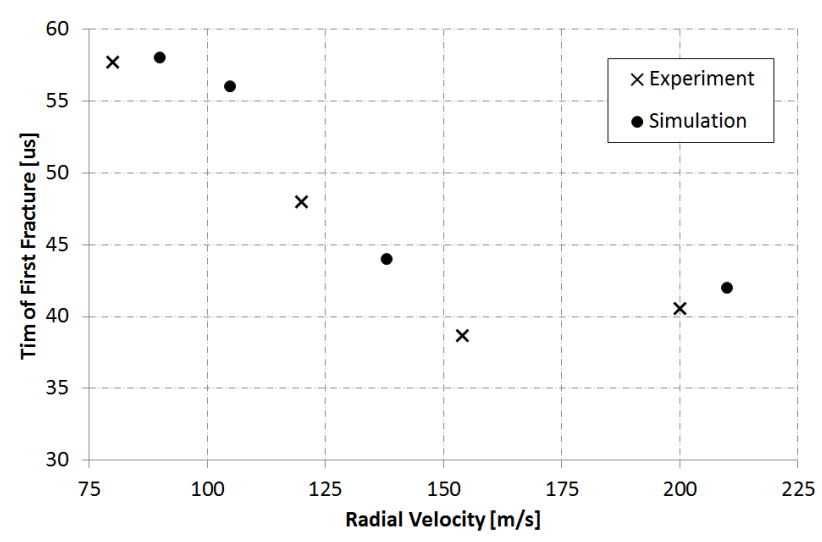

Fig. 19 Comparison of Measured and Simulated Time of First Fracture as function of Radial Expansion Velocity

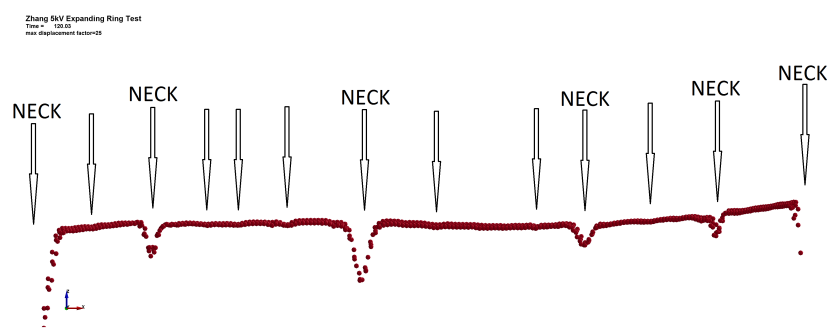

Fig. 20 Top surface of a fragment, axial displacement amplification factor $=25$

\subsection{Number of Necks}

As mentioned in section 6.1 during the simulation a number of necks develop. Some of these necks develop into fractures. Other necks are arrested and bending of some fragments around an arrested neck can be observed. These observations are in agreement with the experimental data. For completeness this section contains the comparison of experimentally measured and simulated number of necks. The experimental results in Zhang and Ravi-Chandar (2006) are based on measuring the variation of thickness of the ring. Hence a similar approach was used for the determination of necks in the simulation results. However, since in the experiments no criterion was provided to determine when a variation in thickness is a neck, only clearly observable necks in the simulation results have been included. To illustrate this a typical top surface obtained in the simulations is shown in figure 20. In order to make it easier to identify thickness variations the dispacement in the axial direction has been amplified by a factor of 25. On this figure variations of thickness are indicated with a arrow and labelled when they were considered a neck. The number of necks in simulations and experiments is summarised in table 6 and Figure 21 for all tests. The number of clearly identifyable necks is
Table 6 Comparison of Measured and Simulated Number of Necks

\begin{tabular}{|c|c|c|}
\hline \multirow[t]{2}{*}{ Test } & \multicolumn{2}{|c|}{ Number of Necks } \\
\hline & Experiment & Simulation \\
\hline $5 k V$ & 17 & 14 \\
\hline $6 k V$ & 20 & 16 \\
\hline $7 k V$ & 29 & 20 \\
\hline $8 k V$ & 32 & 25 \\
\hline
\end{tabular}

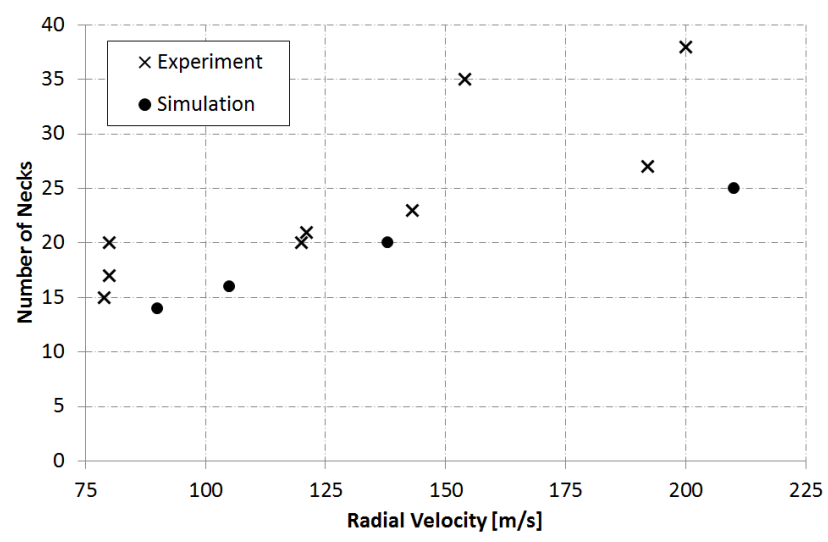

Fig. 21 Comparison of Measured and Simulated Number of Necks

lower than the number of necks reported in Zhang and Ravi-Chandar (2006) but the overall trend of increasing number of necks with increasing expansion speed is well captured. The RMS error is 7.4. The lower number of identified necks in the simulations may be due several factors. Firstly, it may be due to differences in the criterion used to consider a thickness variation as a neck. Secondly, the number of necks depends on hardening sensitivities. The Johnson-Cook model was used in this work because input data was available. Therefore the use of constitutive laws which are more accurate at describing hardening over a range of strain rates and temperatures, for example Rusinek-Klepaczko (Rusinek and Zaera, 2007) or Preston-Tonks-Wallace (Mercier et al., 2010) may provide better agreement with experimental results. In the work by Mercier et al. (2010) it was in fact shown that for pure copper and tantalum the Preston-Tonks-Wallace constitutive equation predicts more necks (as observed in experiments) than simpler constitutive laws such as Zerilli-Armstrong and a power law.

\section{Conclusion}

The modelling of necking and fracture at high strain rates, and in particular modelling of electromagneti- 
cally driven ring experiments has been studied in this paper. It has been demonstrated that a total Lagrangian Smoothed Particle Hydrodynamics method with normalised kernel is a practical discretisation technique for this problem. However, in order to correctly model fracture in this total Lagrangian SPH formulation it was necessary to implement a visibility criterion based on a truncated cone which removes particles from the neighbour list if they are obscured by a failed particle. This method was implemented in 3D and has been demonstrated to be effective and robust. The material behaviour has been modelled with a Johnson-Cook plasticity model in combination with a Lemaitre damage model. The effect of Joule heating due to the current induced in the ring was taken into account in the constitutive model. The electromagnetic and mechanical models are coupled since the electromagnetic force is dependent on the radius of the ring, and the electromagnetic force drives the ring and the constitutive equation is function of temperature which is affected by the Joule heating in the ring. Therefore the loading has been applied by using the measured currents in the ring and by solving a $1 \mathrm{D}$ electromagnetic model and the 3D SPH thermo-mechanical model. Using this modelling approach the number of fragments was predicted with an RMS error of 1.8 fragments and the time at which the first fracture occurs was predicted with an RMS error of $4.9 \mu \mathrm{s}$. In agreement with expermental observations the simulations also predicted the presence of arrested necks and fragments exhibiting bending around an arrested neck. The trend of increase in number of necks with increasing expansion speed is captured, however the number of necks is underestimated in the simulation results with the RMS error of 7.4.

\section{References}

Becker R (2002) Ring Fragmentation predictions using the Gurson model with material stability conditions as failure criteria, Int J Solids Struct, 39, 3555-3580

Belytschko T, Xiao SP (2000) Stability analysis of particle methods with corrected derivatives, Comput Math Appl, 43, 329350

Corbett BM (2006) Numerical simulations of target hole diameters for hypervelocity impacts into elevated and room temperature bumpers, Int J Impact Eng, 33, 431-440

Dong Q, Thomas E, Shaofan L, Wing Kam L (2008) Meshfree simulation of failure modes in thin cylinders subjected to combined loads of internal pressure and localised heat, Int J Numer Methods Eng, 76, 11591184
Fyfe JM, Rajendran AM (1979) Dynamic pre-strain and inertia effects on the fracture of metals, J Mech Phys Solids, 28, 17-26

Grady DE, Benson DA (1983) Fragmentation of Metal Rings be Electromagnetic Loading, Exp Mech, December, 393-400.

Guduru PR, Freund LB (2002) The dynamics of multiple neck formation and fragmentation in high rate extension of ductile materials, Int J Solids Struct, 39, 5615-5632

Hiroe T, Fujiwara K, Hata H, Takahashi H (2008) Deformation and fragmentation behaviour of exploded metal cylinders and the effects of wall materials configuration, explosive energy and initiated locations, Int J Impact Eng, 35, 1578-1586

Hopson MV, Scott CM, Patel R (2011) Computational comparisons of homogeneous and statistical descriptions of AerMEt100 steel subjected to high strain rate loading, Int J Impact Eng, 38, 451-455

$\mathrm{Hu}$ X, Daehn GS (1996) Effect of velocity on flow localisation on tension, Acta Mater, 44 (3), 1021-1033

Johnson JN (1981) Dynamic Fracture and Spallation in ductile solids, J Appl Phys, 52 no. 4 2812-2824

Landen D, Wetz D, Satapathy S, Levinson S (2009) Electromagnetically Driven Expanding Ring With Preheating, IEEE Trans magn, 45 598-603

Lemaitre J, Chaboche JL (1990) Mechanics of Solid Materials, Cambridge University Press.

Lemaitre J, Sermage JP (1997) One Damage Law for Different Mechanisms, Comput Mech, 20, 84-88

LSTC (2007) LS-Dyna Keyword User's Manual, Version 971, Volume I and II, LSTC, Livermore, California.

Meulbroek JP, Ramesh KT, Swaminathan PK, Lennon AM (2008) CTH simulations of an expanding ring to study fragmentation, Int J Impact Eng, 35, 1661-1665

Meyer HW Jr, Brannon RM (2012) A model for statistical variation of fracture properties in a continuum, Int J Impact Eng, 42, 48-58

Meyers MA (1994) Dynamic Behavior of Materials, John Wiley \& Sons, New York.

Mercier S, Molinari A (2004) Analysis of multiple necking in rings under rapid radial expansion, Int J Impact Eng, 30, 403-419

Mercier S, Granier N, Molinari A, Llorca F, Buy F (2010) Multiple necking during the dynamic expansion of hemispherical metallic shells, from experiments to modelling, J Mech Phys Solids, 58, 955-982

Pandolfi A, Krysl P, Ortiz M (1999) Finite Element simulation of ring expansion and fragmentation: The capturing of length and time scales through cohesive models of fracture. Int J Fract, 95, 279-297 
Rabczuk T, Belytschko T, Xiao SP (2004) Stable particle methods based on Lagrangian kernels, Comput Methods Appl Mech Eng, 193, 1035-1063

Rabczuk T, Belytschko T (2004) Cracking particles: a simplified meshfree method for arbitrary evolving cracks, Int J Numer Methods Eng, 61, 2316-2343

Randles PW, Libersky LD (1996) Smoothed Particle Hydrodynamics: Some recent improvements and applications. Comput. Methods Appl Mech Eng, 139, 375-408

Rusinek A, Zaera R (2007) Finite element simulation of steel ring fragmentation under radial expansion, Int J Impact Eng, 34, 799-822

Satapathy S, Landen D (2006) Expanding ring experiments to measure high-temperature adiabatic properties, Int J Impact Eng, 33, 735-744

Shenoy VB, Freund LB (1999) Necking bifurcations during high strain rate extension, J Mech Phys Solids, 47, 2209-2233

Sørensen NJ, Freund LB (2000) Unstable neck formation in a ductile ring subjected to impulsive radial loading, Int J Solids Struct, 37, 2265-2283

Swegle JW, Hicks DL, Attaway SW (1995) Smoothed Particle Hydrodynamics Stability Analysis, J Comp Phys, 116, 123-134

Triantafyllidis N, Waldenmyer JR (2004) Onset of necking in electro-magnetically formed rings, J Mech Phys Solids, 52, 2127-2148

Vadillo G, Rodriguez Martinez JA, Fernandez Saez $\mathrm{J}$ (2012) On the interplay between strain rate and strain rate sensitivity on flow localisation in the dynamic expansion of ductile rings, Int J Solids Struct, 49, 481-491

Vignjevic R, Reveles JR, Campbell J (2006) SPH in a total lagrangian formalism, CMES - Comput Model Eng Sci, 14 (3), pp. 181-198

Vignjevic R, Campbell J, Jaric J, Powell S (2009) Derivation of $\mathrm{SPH}$ equations in a moving referential coordinate system, Comp Methods Appl Mech Eng, 198 (30-32), 2403-2411

Zhang H, Ravi-Chandar K (2006) On the dynamics of necking and fragmentation - I. Real-time and postmortem observations in $\mathrm{Al}$ 6061-O, Int J Fract, 142, 183-217

Zhang H, Ravi-Chandar K (2008) On the dynamics of necking and fragmentation - II. Effect of material properties, geometrical constraints and absolute size, Int J Fract, 150, 3-36

Zhang H, Ravi-Chandar K (2009) On the dynamics of necking and fragmentation - III. Effect of cladding with a polymer, Int J Fract, 155, 101-118

Zhang H, Ravi-Chandar K (2009) On the dynamics of necking and fragmentation - IV., Expansion of $\mathrm{Al}$
6061-O tubes, Int J Fract, 163, 41-65

Zhang H, Ravi-Chandar K (2009) Dynamic Fragmentation of ductile materials, J Phys D: Appl Phys, 42 (21)

Zhou F, Molinari JF, Ramesh KT (2006) Analysis of the brittle fragmentation of an expanding ring, Comput Mater Sci, 37, 74-85

Zhou F, Molinari JF, Ramesh KT (2006) An elasticvisco-plastic analysis of ductile expanding ring, Int J Impact Eng, 33, 880-891 